\title{
A study of static shift removal from magnetotelluric data
}

\author{
D.Beamish, British Geological Survey, Keyworth, Nottingham, NG12 4BX, UK \\ J.M. Travassos, Departamento de Geophysica, CNPq-Observatorio Nacional, R. Gen. Bruce \\ 586, 20291 Rio de Janeiro, Brazil and Petrobras/Cenpes/Segef, 21910 Rio de Janeiro, Brazil \\ Beamish, D. and Travassos, J.M., A study of static shift removal from magnetotelluric \\ data. Journal of Applied Geophysics, 29, 157-178. DOI: 10.1016/0926-9851(92)90006-7
}

\begin{abstract}
Near-surface inhomogeneities can distort magnetotelluric (MT) data, thereby limiting interpretational accuracy. In the specific case of near-surface galvanic distortion the resulting frequency independent shift in apparent resistivity sounding curves can be most readily identified in data which are predominantly one-dimensional ( 1 D). We here consider data from a detailed grid survey which are thoroughly contaminated by parallel offsets. The data were collected over a limited portion of the basalt-covered Paraná basin in Brazil. The data appear largely I D over a three decade bandwidth down to a low frequency limit of about $0.1 \mathrm{~Hz}$. A realistic conceptual model consists of a variable thin-sheet over a basin in which the layer parameters vary sufficiently slowly (laterally) that a I D interpretation of the static corrected data is valid down to some maximum depth (in this case basement). We consider the performance of three methods which attempt to remove static offsets and provide assessments of the configuration of the geoelectric units of the basin. The three methods consist of curve shifting, statistical/spatial averaging, and the application of parameter constraints. The unambiguous removal of static offsets undoubtedly requires one or more independent constraints at each measurement location. In the absence of such control the three methods are necessarily statistical and each must supply a constraint that has least conflict with the data characteristics. Simple spatial averaging of our data proves effective but the degree of success is generally not known. Parametric constraints, applied as uniform layer resistivities, allow phase-only (non-distorted) data inversion. Inverse models obtained from this approach are found to provide accurate estimates of the depth to the main resistivity interface, the base of the basalt cover, in the vicinity of a control well.
\end{abstract}

\section{Introduction}

It is well established that near-surface inhomogeneities of small-scale may complicate the interpretation of magnetotelluric (MT) data. The complications, generally referred to as near-surface distortions, have been studied in theoretical modelling studies (Wannamaker et al., 1984; Park, 1985) and have been well demonstrated in a number of case histories (Jones, 1988; Sternberg et al., 1988).
As noted in a review by Jiracek (1990), any resistivity contrast due to small-scale heterogeneities in the vicinity of the electric field measurements can give rise to a particular class of perturbation referred to as galvanic distortion. The two measured electric fields $\left(E_{x}, E_{y}\right)$ are perturbed from their regional values and a static (frequency.independent) shift of the apparent resistivity sounding curve takes place. The phase between the electric and magnetic fields is unaffected. It is possible, depending on the scale of the feature, that the two electric field components will be perturbed by separate amounts. The parallel shifts that occur in the dual logarithmic coordinates of apparent resis- 
tivity and frequency, if unidentified and uncorrected, would result in incorrect interpretation.

The increasing use of dense and accurate MT measurements has identified the widespread occurrence of static distortion and produced a need for techniques that can successfully correct the distorted data. Since galvanic distortion produces a well-defined perturbation, the basic requirement is to estimate the distortion factors (or site gains) which are two real, frequency-independent numbers operating on the undistorted, regionally-correct electric fields at each sounding site (Larsen, 1977). Although recent schemes to suppress the influence of static effects have involved the use of "continuous" profile measurements (Bostick, 1986; Oldenburg, 1990) only conventional discrete MT soundings are considered here. In theory, knowledge of the near-surface resistivity structure obtained from well-logs should provide the most precise constraints for data normalisation. As far as we are aware, however, the literature provides no examples of resistivity well-log information being used to correct distorted MT data. A deep resistivity well-log for the survey area is presented and is used to provide control in the assessment of the distortion-correction methods. Distortion-correction techniques have been discussed by Jones (1988), Sternberg et al. (1988), and Jiracek (1990). The use of independent inductive geophysical measurements, such as transient EM soundings (Sternberg et al., 1988; Pellerin and Hohmann, 1990), has advantages but cannot assist with the interpretation of many existing data sets. In the absence of such independent control (at each sounding site), it is necessary to assess the intrinsic characteristics of the MT data set for "consistency" and to introduce, if required, some form of sensible constraint. Such assessments are, of necessity, statistical and any constraint takes the place of the unavailable independent control.

Distortion removal techniques that have been considered are (1) curve shifting, (2) statistical or spatial averaging, and (3) parameter constraints (Jiracek, 1990). The successful application of any of these three methods will depend on the data characteristics, particularly the degree of structural complexity. The methods can be most simply applied to data sets which are predominantly one dimensional (1 D). Here the data from a detailed $(2 \mathrm{~km})$ grid survey in the Paraná basin, Brazil are used to compare the performance of the three techniques. The large number of soundings (84) together with the predominantly $1 \mathrm{D}$ characteristics of the data across a limited portion $(28 \times 12 \mathrm{~km})$ of the basalt covered basin provide an almost ideal case. All the soundings, in both electric field components, appear to be influenced by significant parallel offsets.

\section{The well-log control}

The Paleozoic sediments of the basin are covered by the world's most extensive flood basalt complex (Zalán et al., 1986). The 1-2 $\mathrm{km}$ thick basalts (Mid- to Early Cretaceous) cover an area of over $800,000 \mathrm{~km}^{2}$ as shown in Fig. 1a. The underlying basin comprises a complex sequence of conductive $(<25 \Omega \cdot \mathrm{m})$ marine and continental sediments. The preDevonian seismic basement is estimated to be at a depth of about $3 \mathrm{~km}$ in the region of the survey area (Fig. 1a).

The survey area, referred to by locality as Clevelandia, is shown in Fig. 1 b. Deep well-logs are available from well 2-AL-1-SC in the vicinity of sites 8 and 10 . The induction $\log (100-$ $3881 \mathrm{~m}$ ) is shown in Fig. 2. The base of the Serra Geral basalts is the first stratigraphic marker at $1230 \mathrm{~m}$ and beneath this horizon a sequence of conductive sedimentary units occur (Zalán et al., 1986). The well encounters Silurian sediments at $3377 \mathrm{~m}$ and terminates in metagabroic basement ( $3850-3881 \mathrm{~m})$. The high-variance and non-uniform resistivity profile through the flood basalt sequence is a common feature of many well-logs in the Paraná basin. The well-log also reveals a series of thin 

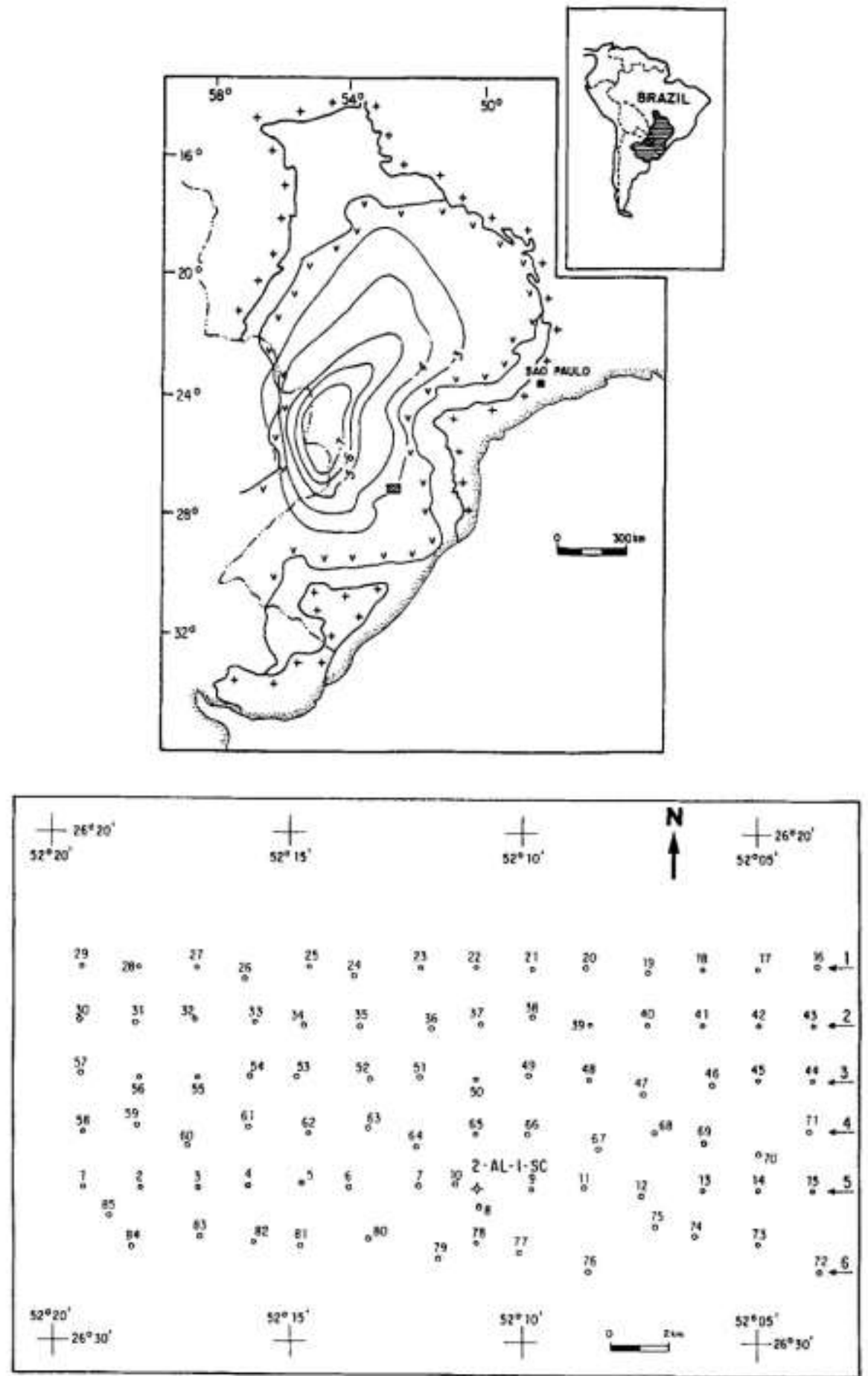

SITE LOCATION MAP CLEVELANDIA DATA

Fig. la. Main location map. Paraná basin outlined on inset map of South America. Main map shows extent of basalt cover ( $v$-symbols) and structural contours of depth to seismic basement in $\mathrm{km}$ (after Zalán et al., 1986). Contour interval is 1 $\mathrm{km}$. Position of Clevelandia MT array is indicated by the solid rectangle (not to scale). b. Sounding locations of the grid array and control well 2-AL-1-SC. No data exists for location 48. Positions of 6 approximately E-W lines are arrowed. 
CLEVELANDIA WELL-LOG 2-AL-1-SC

$4 \& 7$-LAYER INVERSIONS, SITES $8 \& 10$

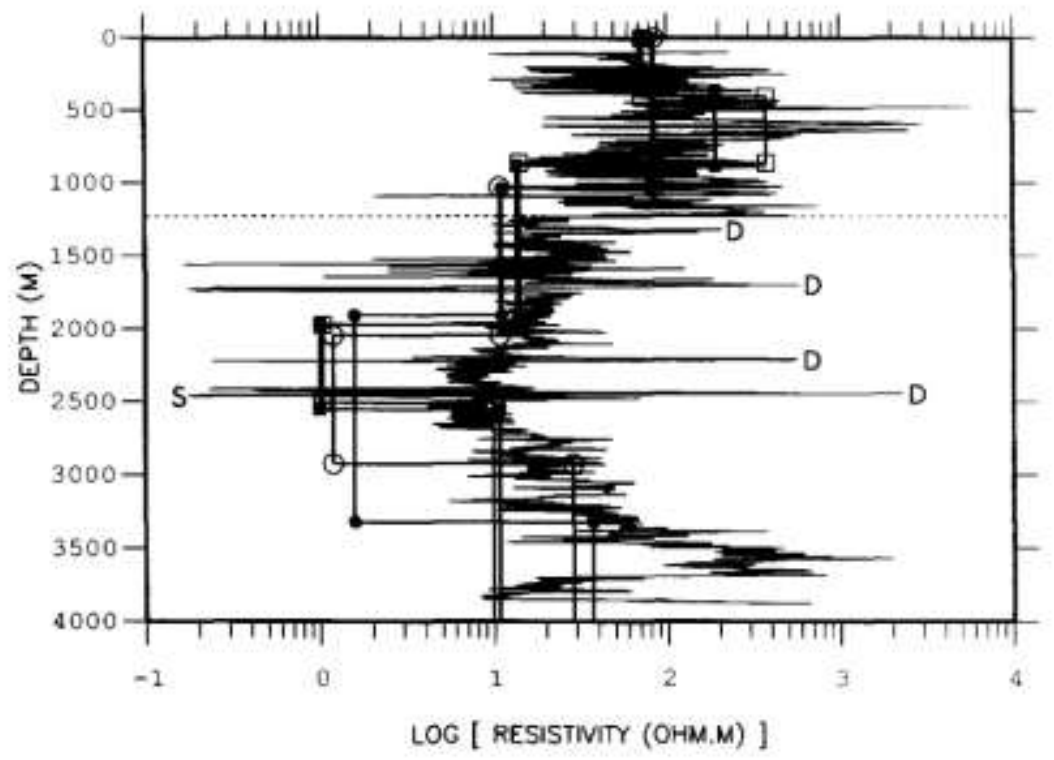

Fig. 2. Induction log from 2-AL-1-SC, located as in Fig. 1b. The first stratigraphic marker at $1230 \mathrm{~m}$ (broken horizontal line) is the base of the Serra Geral flood basalts; $D$ denotes a diabase sill and $S$ a shale rock in the Irati formation. Also shown is a comparison of 4 and 7 layer inverse models (using invariant data, method 2) at sites 8 and 10. Circles denote 4 layer models and squares the 7 layer models. Solid symbols are site 8 and open symbols are site 10.

(maximum thickness $30 \mathrm{~m}$ ) resistive diabase sills which are denoted $D$ in Fig. 2. The lines with symbol corners are layered models which are discussed later.

The induction log at the metric scale clearly monitors high wavenumber variations in the resistivity profile that cannot be resolved by surface soundings. Low wavenumber contributions can, however, be observed particularly in relation to the profile minimum which occurs in the vicinity of the Irati formation which contains an important hydrocarbon source shale ( $S$ in Fig. 2). In terms of interfaces, the only unconformity which gives to a major resistivity transition is the one occuring at the base of the flood basalts.

\section{Data characteristics}

The data discussed here were acquired by a commercial contractor in the early 1980's. Some of the background to MT measurements in the Paraná basin is given by Stanley et al. (1985) who discuss a regional MT survey conducted further north. The 84 grid sounding locations, with site separations of about $2 \mathrm{~km}$, are shown in Fig. Ib.

The data were made available by Petrobrás, the Brazilian national oil company, but they do not comprise the complete MT tensor. The sounding data and associated errors were digitised from paper records and only the main offdiagonal tensor elements are available for analysis. In our estimation the digitisation procedure has not introduced any significant errors into the data set. Although azimuthal (and other tensor decomposition) information cannot be obtained, the assessment and removal of near-surface distortion effects is generally carried out using the two off-diagonal components in the measured (unrotated) coordinate frame. The two off-diagonal impedance elements will be referred to as the $X Y$ component (relating to the measured $E_{x}$ 
electric field) and the $Y X$ component (relating to the measured $E_{y}$ electric field). The Clevelandia data have been previously investigated by Beamish and Travassos (1992a) who describe possible data processing strategies in the context of noise reduction. The data used in the present study are the raw digital data as described above.

An example of the type of data obtained across the array is shown in Fig. 3. The data comprise the $Y X$ components from the 21 locations making up the SW quadrant of the survey grid. The sounding bandwidth covers the five decades from $100 \mathrm{~Hz}$ to $0.001 \mathrm{~Hz}$. In Fig. 3 error estimates are omitted for clarity and the discrete frequency estimates have been joined by continuous lines in order to clarify data "offsets". It is very apparent in Fig. 3 that persistent frequency-independent offsets occur in the apparent resistivity data while the phase data display a high degree of consistency (spa- tial independence). The apparent resistivity data display parallel offsets that range over a decade in the logarithmic scale and nearly all soundings appear to be influenced to some degree.

To illustrate the data characteristics observed across the whole array, and to provide a high degree of averaging (of assumed random levels of distortion offsets ), the 21 soundings in each of the 4 quadrants (i.e. NW, NE, $\mathrm{SE}$ and $\mathrm{SW}$ ) of the complete array were used. The $X Y$ and $Y X$ data components are treated separately and the averaging procedure involves the logarithm of the apparent resistivity values while the phase values are treated in a linear manner at each frequency. Figure 4 displays the averaged $X Y$ component data as continuous lines and the $Y X$ component data as symbols. In Fig. 4 it can be seen that in each component, the quadrant average sounding is a curve whose apparent resistivity appears to

YX COMPONENT DATA

21 SOUNDINGS - SW QUADRANT
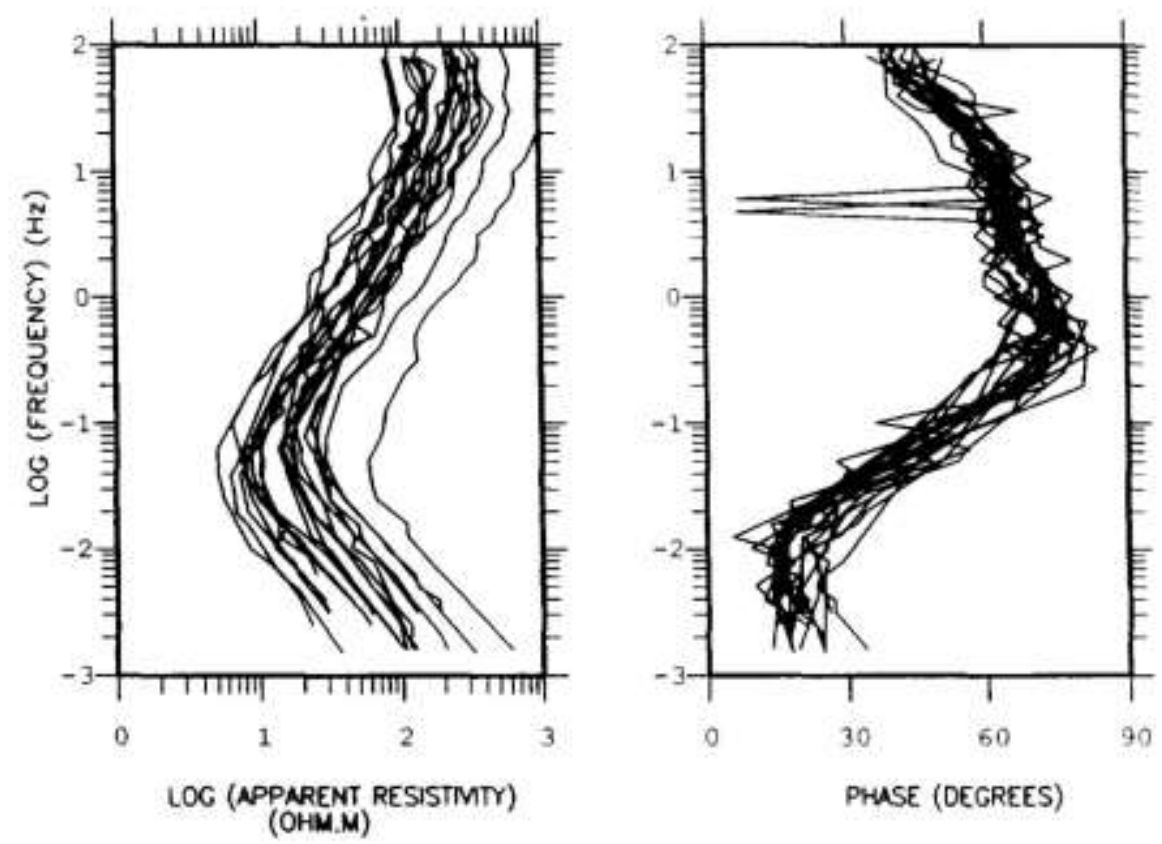

Fig. 3. Data examples. Sounding curves from the 21 locations in the SW quadrant of the array. $Y X$ component data. Discrete frequency estimates have been joined by lines and error bars are omitted for clarity. 


\section{AVERAGED SOUNDINGS - 4 QUADRANTS}

XY-DATA (LINE), YX-DATA (SYMB)
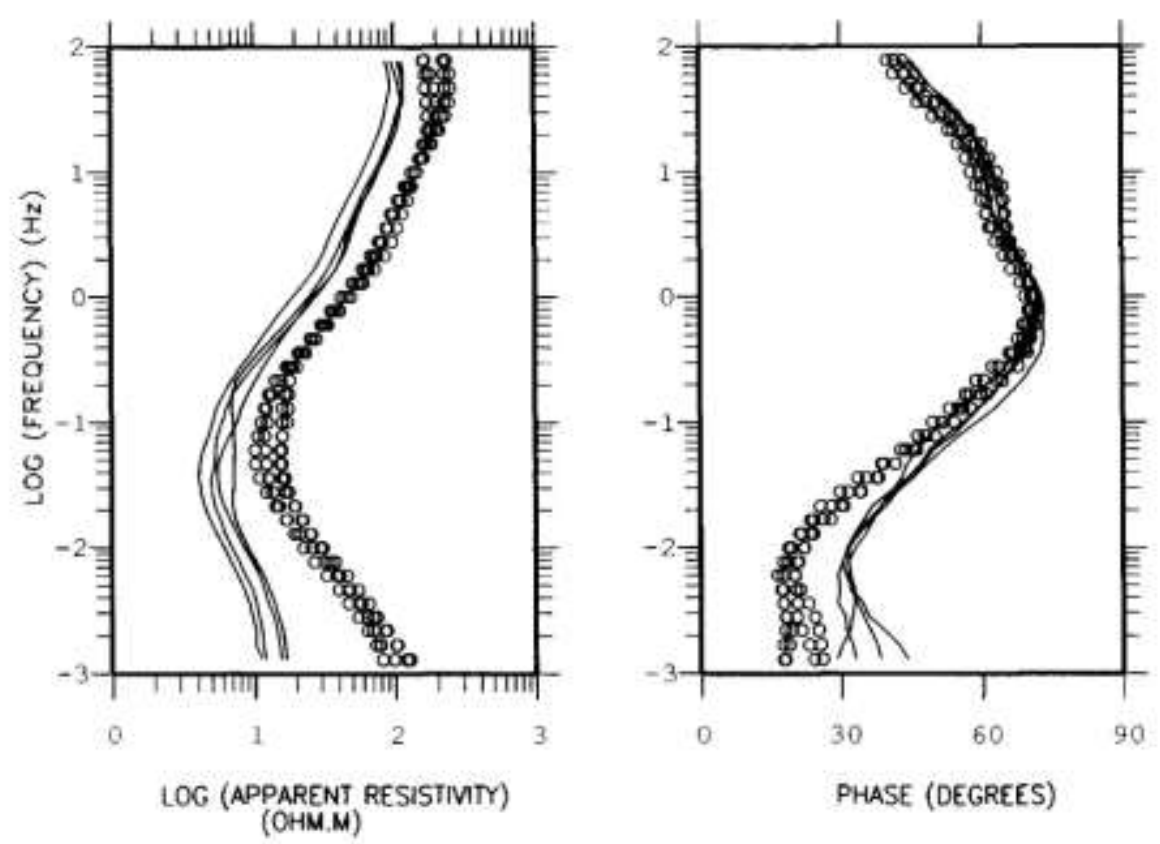

Fig. 4. Spatially-averaged soundings. The 4 averaged soundings use the data in each of the 4 quadrants (NW, NE, SE and $\mathrm{SW}$ ) of the complete array. $X Y$ component data (lines) and $Y X$ component data (symbols).

undergo a moderate amount of parallel shift across the 4 estimates while the phase remains substantially the same until the frequency falls below 1 to $0.1 \mathrm{~Hz}$. At all frequencies a systematic effect is observed in the separation of the $X Y$ and $Y X$ component averages of apparent resistivity. At frequencies below $0.1 \mathrm{~Hz}$ the two components display a gradual divergence in both apparent resistivity and phase.

The systematic parallel offset (i.e. $\rho_{y x}>\rho_{x y}$ at all sites) is unusual. Such behaviour essentially requires that the geological features giving rise to the observed offsets must possess a consistent spatial strike. A simple two-dimensional modelling study of the data (Beamish and Travassos, 1992b) indicates that the data characteristics can be accounted for by a basin structure which is predominantly $1 \mathrm{D}$ but which contains a number of embedded dykes extending to shallow $(0-50 \mathrm{~m})$ depths. For data which display the characteristics shown in
Figs. 3 and 4, particularly the highly consistent phase behaviour in the off-diagonal components, a realistic conceptual model consists of a $3 \mathrm{D}$ thin-sheet over an earth in which the layer parameters vary sufficiently slowly (laterally) that a $1 \mathrm{D}$ interpretation of the static corrected data is valid down to a certain minimum frequency. For our data the minimum frequency is about $0.1 \mathrm{~Hz}$. This form of conceptual model has been discussed previously for MT data acquired across a sedimentary basin by Jones (1988). In our case the structures in the nearsurface sheet appear to provide random offsets in the individual $(X Y$ and $Y X)$ components but a systematic offset such that $\rho_{y x}>\rho_{x y}$ at all sites.

\section{D layered inversion}

Assessments of static distortion can be made in both data and model spaces. According to 
our conceptual model, discussed above, 1 D structural models are appropriate. In 1 D inversion, static distortion shifts all resistivities by a factor equal to the amount of the parallel offset between resistivity curves and inverted depths are shifted by an amount proportional to the square root of the offset (Sternberg et al., 1988). The presence of static distortion essentially leads to excessive structure in crosssectional models determined from the data.

A large number of algorithms exist which solve the 1 D MT inverse problem in terms of a layered model with layer parameters of resistivity and thickness. The different formulations all attempt to minimise the error between the observed data and that of a theoretical "minimum-norm" model. The layered inversion method used here is that presented by Fischer and Le Quang (1981). Other layered inversion schemes (e.g. Jupp and Vozoff, 1975) give essentially the same results when applied to our data. The chi-squared statistic (or its square-root, the RMS misfit) is a common measure of misfit between observed and modelled data. In layered inversion schemes the misfit will be a function of the number of layers which is not known $a$ priori but has to be established. Using the invariant data (method 2, described later) from site 8 as an example, the misfits for a sequence of inversions using 2 to 7 layers are shown in Table 1 . It would seem fairly clear from these results that a 4 layer ( 3 layers +half-space) model is an appropriate choice since it provides a chisquared misfit of 88.6 , close to the expectation level of the data ( $2 \times 46$ frequency estimates).

Inverse models involving 4 and 7 layers using data (as above) from the two sites in the vicinity of the well are shown alongside the induction-log resistivity profile in Fig. 2. The 4 and 7 layer models are denoted by circles and squares, respectively. The base of the basalt sequence (the most well-defined resistivity interface) is shown by the broken horizontal line. The 4 layer solutions model the basalt as a single layer while the 7 layer solutions provide a 2
TABLE 1

Chi-squared and RMS misfits obtained from successive layered inversions of the invariant (method 2) sounding data at site 8. Starting model in each case is a profile of resistivity $50 \Omega \cdot \mathrm{m}$. Data consists of 46 frequency estimates i.e. 92 data points

\begin{tabular}{lll}
\hline $\begin{array}{l}\text { No. of } \\
\text { layers }\end{array}$ & $\begin{array}{l}\text { Chi-squared } \\
\text { misfit }\end{array}$ & RMS misfit \\
\hline 2 & 1194.3 & 3.60 \\
3 & 1183.2 & 3.59 \\
4 & 88.6 & 0.98 \\
5 & 73.8 & 0.89 \\
6 & 73.4 & 0.89 \\
7 & 83.4 & 0.89 \\
\hline
\end{tabular}

layer sequence which is more consistent with the non-uniform behaviour of the well-log profile. Realistic vertically-averaged resistivity values are returned by both 4 and 7 layer solutions, particularly within the upper $400 \mathrm{~m}$ but the model estimation of the depth to the base of the basalt is poor in all cases. At this point it should be noted that the data used to construct the inverse models shown in Fig. 2 are just one of a set of possible corrected estimates which are discussed later. The resistivity interface at the base of the basalts does however provide a useful form of control when assessing the performance of the correction procedures considered. This control can only be exercised sensibly at sites 8 and 10 .

It can also be noted in Fig. 2 that there is a clear discrepancy between the values of resistivity determined by the inverse models and those returned by the induction tool through the region associated with the minimum resistivities of the section (e.g. layer 3 of the 4 layer models). This "effect" occurs repeatedly in other regions of the Paraná basin (i.e. in other well-logs with associated MT data). According to our investigations the effect is not dependent on the parameterisation of the vertical profile (e.g. layered or smooth) neither is it a residual effect of static distortion. There are a number of physical reasons why such a depthdependent offset may occur and they are cur- 
rently under investigation. For the purposes of the present study the effect is simply noted.

If we accept that a 4 layer representation of the structural units is optimum for our data then from Fig. 2 it can be noted that the basalt sequence is defined by a single layer. The underlying sequence of sedimentary units is then represented by two layers. The upper sequence of Paleozoic sediments has a resistivity of about $10 \Omega \cdot \mathrm{m}$ while layer 3 represents the most conductive unit of the basin and is modelled as having a resistivity of order $1 \Omega \cdot \mathrm{m}$. The underlying half-space, taken to represent electrical basement, occurs at an approximate depth of $3 \mathrm{~km}$ in accord with regional estimates of the depth to seismic basement (Fig. la).

Due to the spatially-consistent nature of the sounding data (Figs. 3 and 4), a 4 layer inverse modelling is applied consistently to all the data. In the model assessments that follow it is important to bear in mind their basic resolution attributes. One of the most useful methods when examining parameter resolution is the $V$ matrix (Jupp and Vozoff, 1975; Raiche et al., 1985) which relates an eigenparameter decomposition to the physical parameters of a particular model. Table 2 shows the eigenparameter $(E P)$ analysis of the 4 layer model at site 8 (Fig. 2). In Table 2 the columns correspond to eigenparameters and the rows correspond to the physical parameters of resistivity $(r)$ and thickness $(t)$. Since each of the eigen- parameters is a linear combination of the logarithms of the physical parameters the resolution of different aspects of the model can be investigated.

In Table 2 the largest contributions to EPI (column 1) are made by the resistivity and thickness of layer 3 . The important parameter contribution is therefore $r_{3} / t_{3}$ i.e. the conductance of the most conducting layer that is resolved. The eigenparameters also indicate that both the resistivity and thickness of layer 1 ( $E P 3$ and $E P 2$ respectively) together with the resistivity of layer 2 (EP6) are resolved individually. The EP4 and EP5 columns indicate that the parameter combinations $r_{4} / t_{2}$ and $r_{4} t_{2}$ are resolved and since both quotient and product are known for the same combination both $r_{4}$ and $t_{2}$ are known individually. The analysis indicates that both the resistivity and thickness of layer 1 (i.e. the basalt) are highly and individually resolved.

In order to assess the three methods using the results of $1 \mathrm{D}$ inversion, model results are compared using either the complete data set or a subset of the array which forms an E-W profile. The results using the complete data set are intended to display the overall statistics while the results along line 5 ( sites $1-15$; Fig. $1 \mathrm{~b}$ ) are intended to demonstrate, in more detail, the spatial variation of the parameters determined. It should be noted that the "true" variation of the geoelectric units across the array is

\section{TABLE 2}

$V$ matrix eigenparameter $(E P)$ analysis for 4 layer, best-fitting inverse model solution. All 7 eigenparameters are significant. Solution obtained using invariant (method 2 ) sounding data at site 8 . Rows correspond to model parameters (logarithmic scale) of resistivity $(r)$ and thickness $(t)$ with subscripts denoting layer number

\begin{tabular}{|c|c|c|c|c|c|c|c|}
\hline & $E P 1$ & $E P 2$ & $E P 3$ & EP4 & EPS & EP6 & $E P 7$ \\
\hline $\log r_{1}$ & 0.001 & 0.046 & 0.989 & -0.101 & 0.066 & -0.069 & 0.005 \\
\hline $\log t_{1}$ & 0.177 & 0.934 & -0.005 & 0.138 & -0.084 & 0.263 & -0.023 \\
\hline $\log r_{2}$ & 0.158 & 0.224 & -0.067 & 0.068 & -0.058 & -0.949 & 0.112 \\
\hline $\log t_{2}$ & -0.026 & 0.177 & -0.125 & -0.662 & 0.684 & -0.067 & -0.206 \\
\hline $\log r_{3}$ & 0.696 & -0.117 & -0.016 & -0.280 & -0.012 & 0.045 & 0.634 \\
\hline $\log t_{3}$ & -0.632 & 0.148 & -0.018 & 0.034 & 0.190 & 0.008 & 0.736 \\
\hline $\log r_{4}$ & 0.245 & -0.090 & 0.027 & 0.670 & 0.694 & 0.026 & 0.018 \\
\hline
\end{tabular}


unknown except in the vicinity of the well. The majority of assessments made are necessarily statistical. When assessing the processing methods via the performance of the variance, or spatial stability, of the inverse model parameters we are tacitly assuming that the simplest hypothesis may be closest to the truth. The simplest hypothesis is that the true structure is the one closest to the $1 \mathrm{D}$ conceptual model. In such circumstances, following removal of static distortion, the "best" models will display minimum variance and spatial stability.

\section{Methods for the removal of static shift}

\section{Individual curve shifting - method I}

The use of inductive controlled source EM measurements to provide an independent control on the non-distorted resistivity at shallow depths was noted previously. The method constitutes a high frequency form of curve shifting at individual sounding locations. In the absence of independent geophysical measurements the statistical behaviour of the data characteristics can be invoked. Park (1985) discusses a $3 \mathrm{D}$ modelling study in relation to the frequency characteristics observed across an 11 site MT survey in Nevada. Parallel shifts observed in the data $(f>1 \mathrm{~Hz})$ were suppressed by curve shifting to one of two (depending on site) "representative" values at 100 $\mathrm{Hz}$. The shifting procedure, referred to as removal of the surface heterogeneity, was applied jointly to the maximum and minimum (i.e. rotated) sounding data. Other examples of individual curve shifting have been presented by Berdichevsky et al. (1989).

An example of the apparent resistivity data in the $Y X$ component from the 21 sites forming the SW "quadrant" of the array was shown in Fig. 4. Figure 5 displays these same data which have been normalised (curve shifted) alongside the corresponding (but unnormalised) phase data. Although $\rho_{y x}>\rho_{x y}$ at each site
(Fig. 4) both components have been normalised to an apparent resistivity value of $34 \Omega \cdot \mathrm{m}$ at $1 \mathrm{~Hz}$ in Fig. 5. The normalisation value ( 34 $\Omega \cdot \mathrm{m}$ ) is simply the median value of all 84 apparent resistivity sounding curves (both $X Y$ and $Y X$ components) at $1 \mathrm{~Hz}$.

The frequency chosen for the normalisation obviously has some physical significance in relation to the structure being probed. Theoretically in a strict $1 \mathrm{D}$ environment subject to galvanic distortion, the sounding data at all frequencies (actually below some very high frequency limit) will display simple parallel offsets. In such a case the choice of normalisation frequency is arbitrary. In the practical case using data with a certain degree of noise, it is advantageous to choose a suitable "stable" frequency. In the case of the present data frequencies below $0.1 \mathrm{~Hz}$ are avoided because of the frequency-dependent anisotropy (the gradual divergence between $\rho_{x y}$ and $\rho_{y x}$ ) that occurs at low frequencies. It is adviseable also to avoid frequencies where turning points exist in the apparent resistivity curves. A choice of $1 \mathrm{~Hz}$ produces the normalised data shown in Fig. 5. The normalisation clarifies the nature of the low frequency anisotropy and performs adequately at high frequencies. It can be seen that over the bandwidth from 100 to $0.1 \mathrm{~Hz}$ the degree of any "residual offset" in the levels observed in the $\rho_{x y}$ and $\rho_{y x}$ components is small. Non-parallel behaviour in both components can be observed over the same bandwidth however. Such behaviour could be noise related but there is no reason to suppose that the behaviour could not be accommodated by slight structural variations over the "sub-array" dimensions of $14 \times 6 \mathrm{~km}^{2}$.

The effects of such curve shifting can be assessed by performing data inversion on the normalised data. The results of 4 layer inversion applied to the 15 soundings from $\mathrm{E}-\mathrm{W}$ line 5 (sites 1 to 15; Fig. 1b) are overlaid in Fig. 6. The results applied to the $X Y$ and $Y X$ components are shown separately and a logarithmic depth scale is used. When examining the re- 

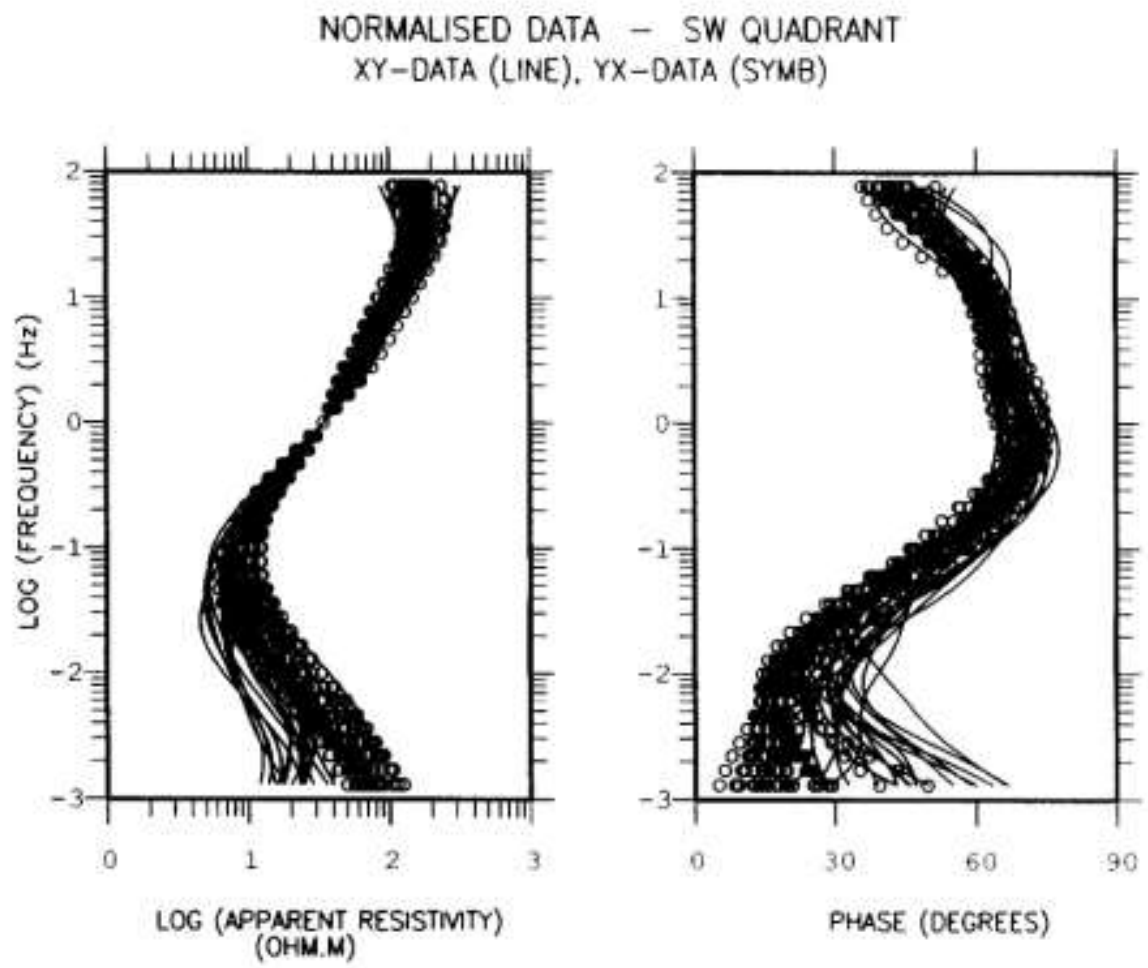

Fig. 5. Example of curve shifting. Apparent resistivity sounding curves from the 21 sites in the SW quadrant have been normalised to the same value of $34 \Omega \cdot \mathrm{m}$ at $1 \mathrm{~Hz}$. The phase data are not included in the normalisation. Solid lines are $X Y$ component data and discrete symbols are $Y X$ component data.

sults shown in Fig. 6 it should be noted that the data traverse a profile of some $28 \mathrm{~km}$ and some structural variations should be allowed for. In Fig. 6 all model parameters display a degree of variance; some of the higher variance features (outliers in Fig. 6) are likely to be due to high levels of measurement noise. Some of the lower levels of variance undoubtedly occur due to parameter trade-offs. The largest difference in the inverse models produced by the $X Y$ and $Y X$ component data occurs in the definition of layer 4 which is a half-space representing the electrical basement. The differences observed in the models obtained from the two components are directly attributable to the low frequency anisotropy illustrated in Fig. 5. According to the discussion of parameter resolution the resistivity and thickness of layer 1 should be the best resolved feature of these 4 layer inversions; this is apparent in the results presented.

\section{Statistical averaging - method 2}

The inhomogeneities giving rise to distortion effects can be viewed as a form of geological noise since they cannot be resolved except by survey methods involving exceptional spatial coverage and/or bandwidth. As noted by Jiracek (1990) the application of filter theory to remove or suppress the undesired effects is natural. Relatively simple spatial averaging of data from closely spaced sites has been described by Sternberg et al. (1985) and Beamish (1990).

The quadrant spatial averages of the array data were shown in Fig. 4 and discussed previously. Although such gross spatial averages are informative, it is also possible to average the soundings with a minimum of spatial overlap to preserve a higher degree of spatial independence. In the case of the present array survey, and with the exception of edge locations, 


\section{4-LAYER INVERSION RESULTS}

LINE 5 (NORMALISED XY,YX DATA)
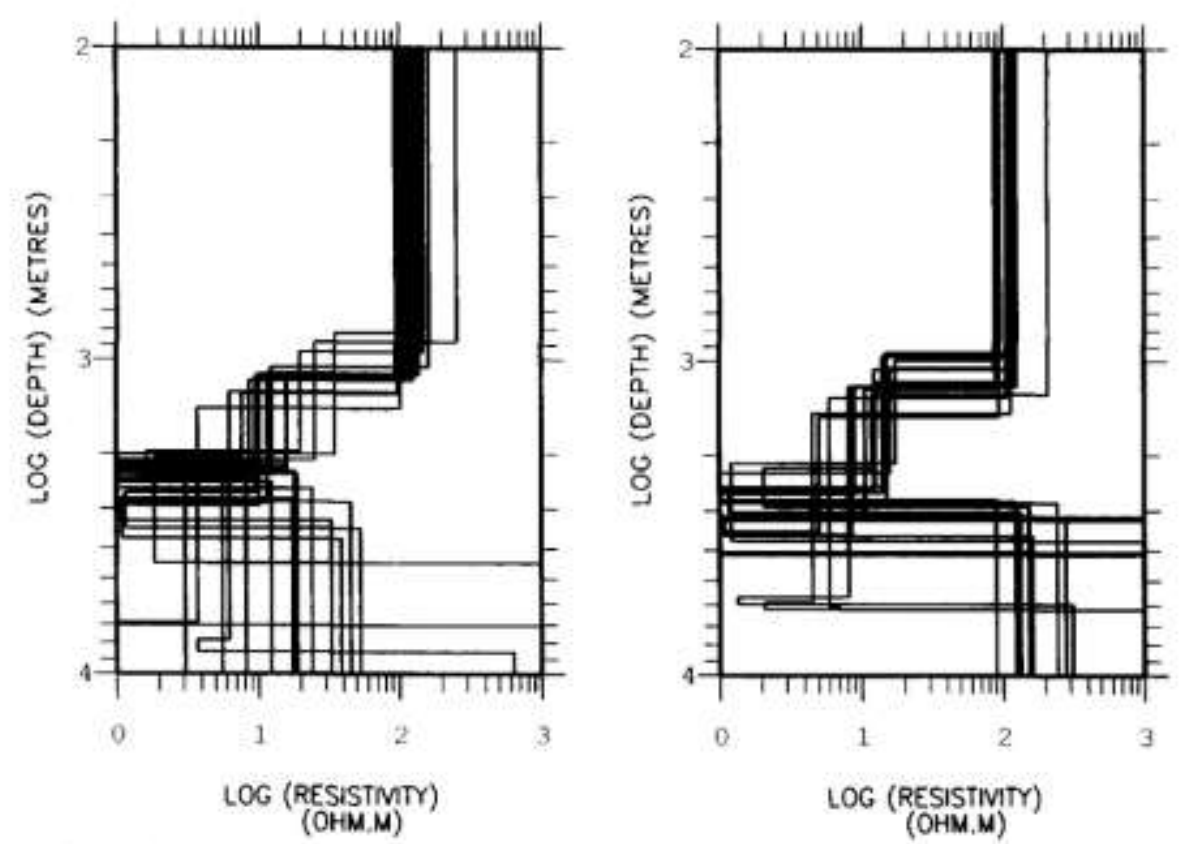

Fig. 6. Examples of best-fitting models obtained following curve shifting of apparent resistivity sounding curves. Results from 15 sites along E-W line $5(28 \mathrm{~km})$ are shown. Left diagram shows $X Y$ component results and right diagram shows $Y X$ component results.

each sounding site has 4 nearest neighbours which form a diamond geometry around the centre site (Fig. 1b). The spatial averaging which involves the minimum of spatial overlap is therefore based on 5 site (diamond) averaging with the average value being assigned to the centre location. Around the edges of the array the averaging function reduces to 4 site (half diamond) configuration. At corner locations only 3 sites can be used. If we assume a completely regular grid with a site separation of $2 \mathrm{~km}$ then diamond averaging provides truly independent averages at centres spaced $6 \mathrm{~km}$ apart. In practice, the average at each centre is provided by a different (unique) set of 5 (or 4) soundings. As previously, the $X Y$ and $Y X$ data components are treated separately and standard errors are obtained using the variance of the estimates included in the average. Obviously a number of different filtering or weighting schemes could be adopted. However, on the basis of the observed data characteristics the distortion level is assumed random in the individual $X Y$ and $Y X$ components and only simple averages (as before, the logarithm of apparent resistivity and linear phase ) are taken i.e. no spatial or frequency-dependent weights are applied.

An effective way to examine the results of the above smoothing operation is to display the apparent resistivity and phase pseudo-sections along a profile. Figure $7 \mathrm{a}, \mathrm{b}$ shows the apparent resistivity (logarithmic scale) and phase data in the $Y X$ component along $\mathrm{E}-\mathrm{W}$ line 5 (sites 1 to 15 ; Fig. 1) for the bandwidth from 100 to $0.01 \mathrm{~Hz}$. In the case of the apparent resistivity data, the vertical zoning caused by varying levels of static distortion is dearly suppressed by the diamond averaging procedure. In the case of the phase data, which are not subjected to 

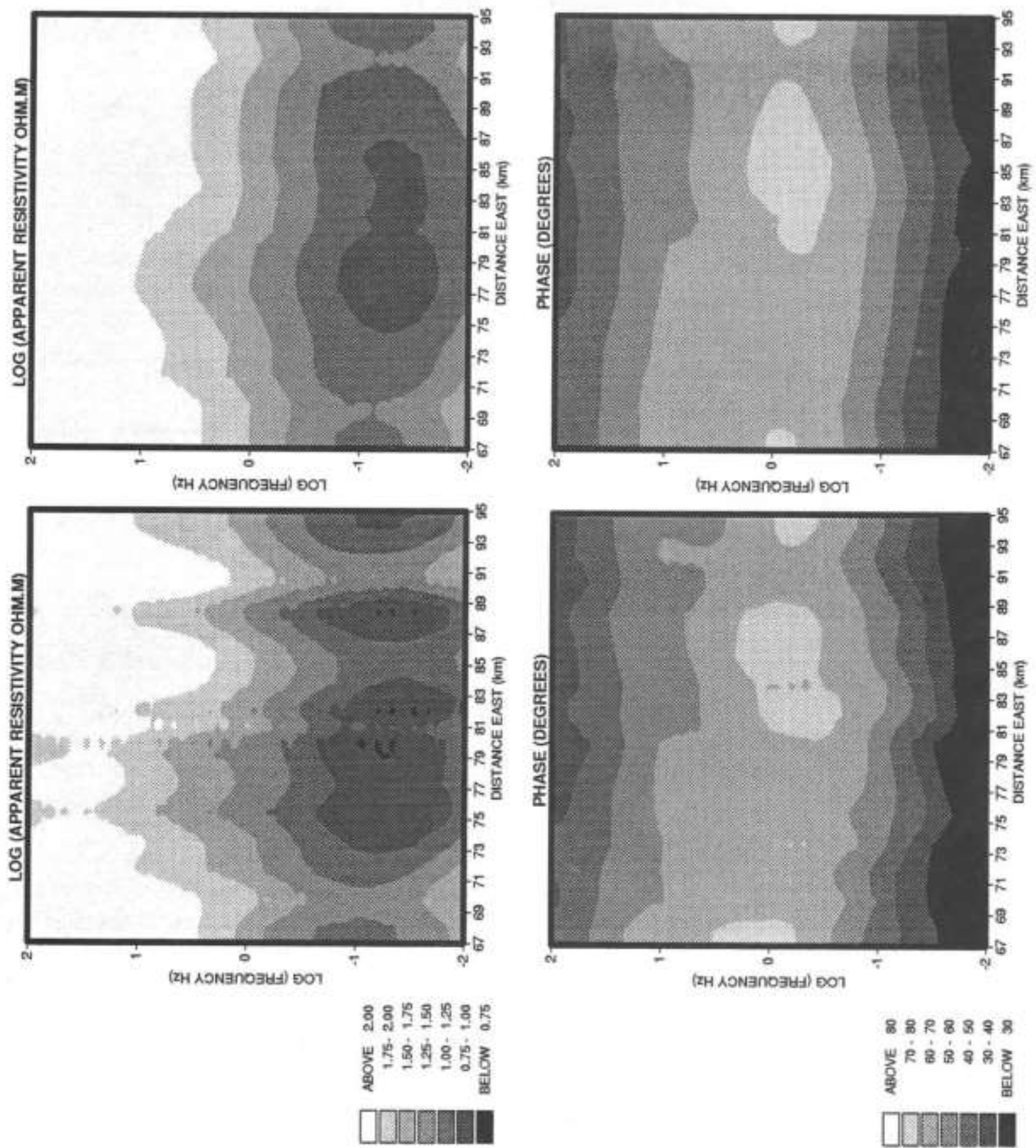

Fig. 7. Comparison of data prior to and following diamond averaging. Data used are the $Y X$ component soundings along E-W line $5(28 \mathrm{~km})$ and are displayed as pseudo-sections in log (apparent resistivity) and phase over 4 decades from 100 to $0.01 \mathrm{~Hz}$. a. Left diagrams show original data. b. Right diagrams show diamond averaged data for the same profile. 

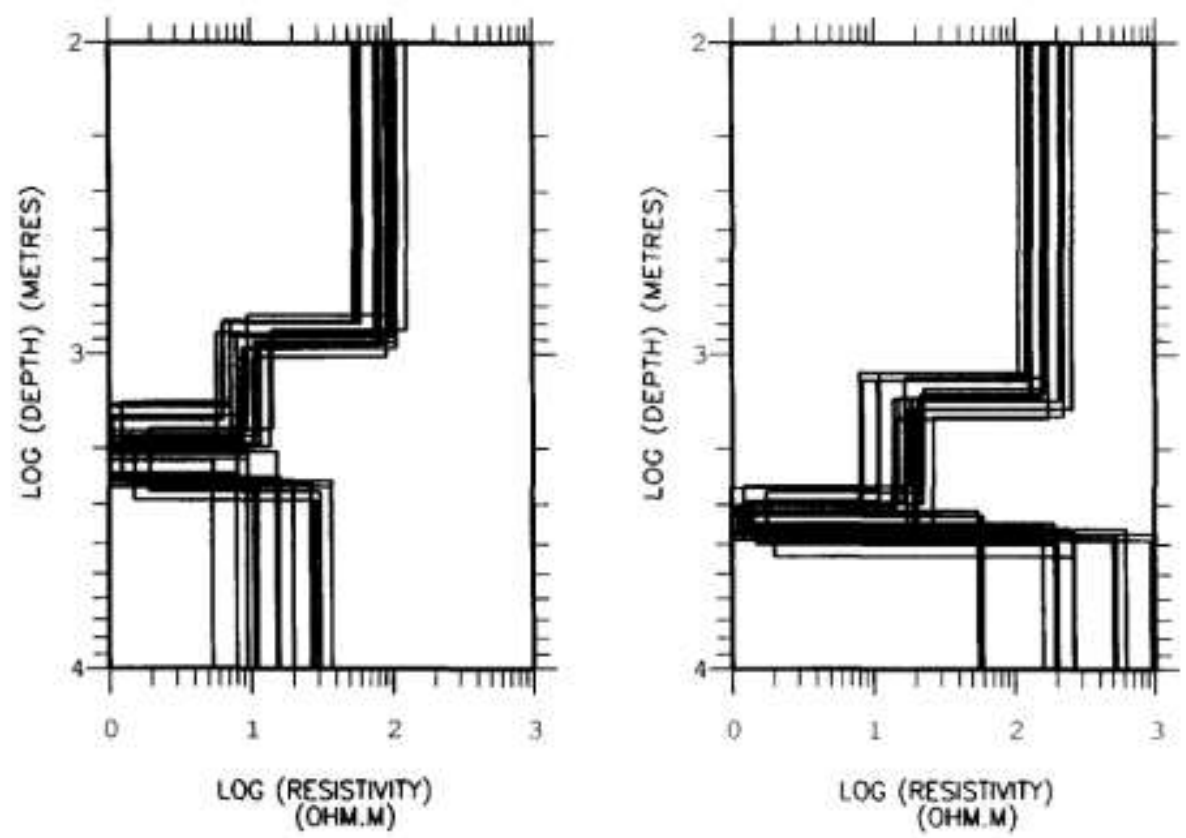

Fig. 8. Examples of best-fitting models obtained following diamond averaging. Results from 15 sites along E-W line 5 ( 28 $\mathrm{km}$ ) are shown. Left diagram shows $X Y$ component results and right diagram shows $Y X$ component results.

distortion effects, the diamond averaging procedure produces a simple lateral smoothing which appears to provide a useful form of noise reduction.

As with the previous method the performance of the processing procedure can be assessed by performing data inversion on the processed data. The results of 4 layer inversion applied to the 15 soundings along line 5 are overlaid in Fig. 8. The results applied to the $X Y$ and $Y X$ components are shown separately and a logarithmic depth scale is used. When compared with the equivalent results of the previous method (method 1, Fig. 6), it is apparent that method 2 has resulted in a greater degree of uniformity in the inverse models obtained along this profile. A greater degree of noise reduction, inherent in the statistical averaging of method 2, appears to produce inverse models with fewer profiles that could be classified as outliers and much reduced vari- ances. This is to be expected given the spatial averaging involved. A closer examination of the behaviour of the models in the individual $X Y$ and $Y X$ components reveals a consistent offset "slope" of $2: 1$ in the dual logarithmic coordinates. The observed behaviour is consistent with the systematic parallel offset between the $X Y$ and $Y X$ component data (Larsen, 1977). Since both $X Y$ and $Y X$ component data appear as statically-shifted versions of each other down to a frequency of about 0.1 $\mathrm{Hz}$ it appears appropriate, and consistent with the conceptual $1 \mathrm{D}$ model, to undertake assessments of the vertical structure using a rotational invariant. For our data we are necessarily restricted to the Berdichevsky invariant which is half the difference of the off-diagonal impedance elements (Berdichevsky and Dmitriev, 1976).

The formation of the invariant sounding curves from the diamond-averaged compo- 

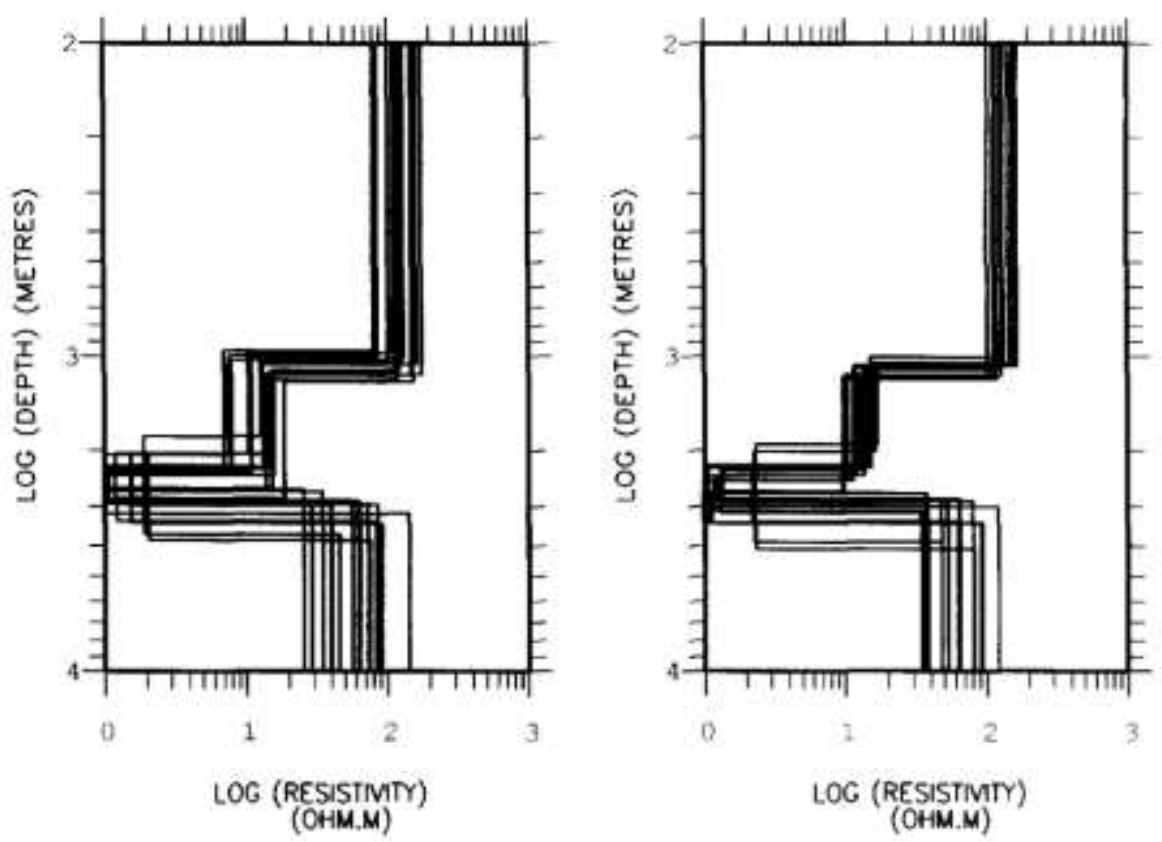

Fig. 9. Examples of best-fitting models obtained following diamond averaging and then forming the rotational invariant. Results from 15 sites along E-W line $5(28 \mathrm{~km})$ are shown. Right diagram shows the results obtained when these same data are further normalised (curve shifted) as in method 1.

nent curves at each site produces a new data set that can be assessed using 4 layer inversion. The results of 4 layer inversion for the invariant data across line 5 are assembled in Fig. 9a and can be compared with the equivalent results obtained using the two individual components (Fig. 8). The comparison indicates that the use of the invariant data has not decreased the variance of the model parameters across the profile. The models determined using the invariant data merely provide the model "average" that follows from the definition of the invariant.

Since the spatial averages were successful in reducing scatter in the inverse model parameters it is instructive to examine the performance of curve-shifting on the processed data. In keeping with the above discussion this was undertaken using the invariant data determined from the diamond-averaged compo- nent data. The normalisation value and frequency were identical to those used in method $1(34 \Omega \cdot \mathrm{m}$ at $1 \mathrm{~Hz})$. The results of the standard 4 layer inversion across line 5 for the normalised data are shown in Fig. $9 \mathrm{~b}$ alongside the equivalent results obtained from the unnormalised data. It is clear from Fig. 9 that this final processing procedure has produced inverse models with a decrease in parameter variance. This is particularly true of the "best-resolved" features of the 4 layer model namely the resistivity and thickness of layer 1 together with the resistivity of layer 2 .

It should be noted that the method of curveshifting adopted here is very restrictive. The use of the same value of apparent resistivity at a single frequency across the whole array actually relies on the conceptual model and effectively assumes that a uniform layer underlies the survey region, at a fixed depth. Since 
the response due to such a layer will encompass a finite bandwidth, the single frequency restriction may not be ideal. The concept of the uniform layer may be better approached using parametric assessments of the inverse model behaviour as discussed below.

\section{Parameter constraints - method 3}

As noted previously, Jones (1988) discusses an a priori assumption that may be used as a constraint in the removal of static shift effects observed across a sedimentary basin. The assumed conceptual model consists of a random $3 \mathrm{D}$ thin-sheet over an earth in which all layer parameters vary sufficiently slowly that a $1 \mathrm{D}$ interpretation of the static corrected data is valid down to a certain frequency. The basic assumption is that at least one layer parameter can be described in a simple parametric manner and that our estimates of that parameter vary in some statistical manner about the true value, due to static distortion. If the parametric behaviour can be established using the bestfitting model estimates, the behaviour can be used as a constraint to normalise the various levels of distortion encountered in the data.

Parameter constraints have been used in layered model construction since the advent of inversion methods. Conventionally, the introduction of a "known" model parameter is equivalent to providing perfect resolution of that parameter in the inverse procedure. In terms of the resolution matrix discussed previously, the fixed parameter cannot enter into parameter equivalencing and as a consequence the resolution of the other model parameters is likely to be enhanced.

Under the quasi $1 \mathrm{D}$ assumption the true resistivity and depth to base of the $i$ th layer are $\rho_{i}(x, y)$ and $d_{i}(x, y)$. Best-fitting $1 \mathrm{D}$ models must be obtained for both off-diagonal components since different distortion factors may operate independently on the measured electric fields $E_{x}$ and $E_{y}$. For the $X Y$ component data the model estimates of the $i$ th layer are

$$
\begin{aligned}
& \rho_{i, x y}(x, y)=\rho_{i}(x, y) \cdot D_{x}^{-2}(x, y) \\
& d_{i, x y}(x, y)=d_{i}(x, y) \cdot D_{x}^{-1}(x, y)
\end{aligned}
$$

(Larsen, 1977) where $D_{x}(x, y)$ is the static shift factor (a real number) for location $(x, y)$ and operates on the electric field $E_{x}$. Similar relationships for the $Y X$ component data provide $D_{y}(x, y)$ which is the static shift factor operating on the electric field $E_{y}$. Jones (1988) also notes that the layer estimates will be interrelated such that, for each layer, the following expression must hold:

$$
\left(d_{i, x y} / d_{i, y x}\right) \cdot\left(\rho_{i, y x} / \rho_{i, x y}\right)^{1 / 2}=1
$$

where the dependence on $(x, y)$ is assumed. The above ratio was determined from the 4 layer inversions at all 84 sites for the first 3 layers. In the case of layer 1 (the best resolved) the median of the distribution (over the 84 results) is 1.079 with an average deviation of 0.162 indicating the "model" is correct.

Statistical descriptions of the behaviour of the model parameters across the array were obtained by performing inversions on a number of data sets. When applied to the original $X Y$ and $Y X$ component data, the models obtained possess high levels of variance (similar to the behaviour of the method 1 data of Fig. 6 ). For this reason our preferred method uses the diamond-averaged (but unnormalised) data of method 2. Thus the data set used has already been processed to provide a high degree of suppression of random distortion effects and the sounding data are not strictly independent. The statistical descriptions were carried out on both $X Y$ and $Y X$ components individually and using the invariants derived at each location. For the data considered here, and excluding the behaviour of the resistivity of the lower half-space (layer 4 ), the results using the invariant provide a summary of the behaviour observed in the models obtained using the individual components.

The results of 4 layer inversion applied to all the diamond-averaged invariant data are 
shown in Fig. 10. The resistivities (Fig. 10a) and depths (Fig. 10b) of layers 1,2 and 3 are identified by different symbols and are plotted as a function of radial distance to indicate spatial dependence. The resistivities of the three layers are well separated with those of layers 1 and 2 displaying the least variance. When examining the model depths it should be noted that the inverse model solutions involve thickness and therefore errors in the depths (to layer base) accumulate with depth. Thus in Figure $10 \mathrm{~b}$, the depth of layer 1 appears spatially stable but the depth estimates of the base of layers 2 and 3 are subject to considerable scatter.

Static distortion (when present) will introduce a constant offset (i.e. due to $D_{x}, D_{y}$ ) in our depth estimates of all three layers at any sounding location $(x, y)$. The increasing variance of all parameter estimates with depth in Fig. 10 is more a characteristic limitation of layered inversion than of the presence of static distortion. For reasons previously discussed, the best statistical estimates will be obtained using the parameters of layer 1 . An examination of the layer 1 parameters suggests that the spatial averaging involved in the formation of the diamond-averaged data has produced a high degree of suppression of distortion offsets. The question remaining is whether a further parametric constraint, applied either to the raw or diamond-averaged data, can improve on the estimates shown in Fig. 10.

In the case of array data a quasi $1 \mathrm{D}$ layer must be represented by a surface in $(x, y)$. As far as we are able to judge, the parametric constraint most appropriate to the type of behaviour observed in the best fitting model estimates is that one or more layers are uniform across the survey region. Under this assumption the variance of the parameters of a given layer, as in Fig. 10, would be due to either distortion offsets in the apparent resistivity sounding data and/or the limitations of the inversion procedure.

In terms of structural assessments of the basin, the least restrictive way to apply a uni- form layer constraint is to fix only the resistivity of that layer and allow the thicknesses (depths) of all layers to be determined. A further advantage in constraining only the resistivity of one or more layers is that inversion algorithms can be applied which determine best-fitting models using the phase data alone. In these circumstances, the apparent resistivity data, which may contain unknown distortion offsets, are not included in the assessment of the best-fitting model solutions and the fixed resistivity of a particular layer provides a spatially-uniform normalisation of these data. This form of inversion exploits the dispersion relationships which connect apparent resistivity and phase in the $1 \mathrm{D}$ case (Weidelt, 1972) and which allow an apparent resistivity sounding curve to be determined from the phase data apart from a constant of normalisation (Clay and Hinich, 1981).

In practice a large number of constrained inversion experiments were performed using both joint apparent resistivity/phase and phase alone. In general the constrained inversions using phase alone gave the most stable depth estimates for each of the three layers. Figure 11 shows the depth estimates obtained from two constrained (layer resistivity) inversions of the diamond-averaged invariant data, using phase alone. The estimates can be compared with the unconstrained estimates of Fig. 10b which were obtained using apparent resistivity and phase jointly. In Fig. 11 a the resistivity of layer 1 was fixed at $125 \Omega \cdot \mathrm{m}$ which is the median value of the distribution shown in Fig. 10a. In Fig. 11b the resistivities of all three layers were constrained to be uniform by assuming median values of $125 \Omega \cdot \mathrm{m}$ (layer 1), $14.452 \Omega \cdot \mathrm{m}$ (layer 2), and $1.19 \Omega \cdot \mathrm{m}$ (layer 3 ).

When assessing the various depth estimates shown in Figs. 10 and 11 for possible variance due to static distortion it is important to compare like-with-like. The introduction of fixed parameters is likely to have increased the resolution of the non-constrained parameters. The precise outcome is difficult to predict since the 


\section{4-LAYERED INVERSION RESULTS \\ LAYER RESISTIVITIES 1.2 \& 3}

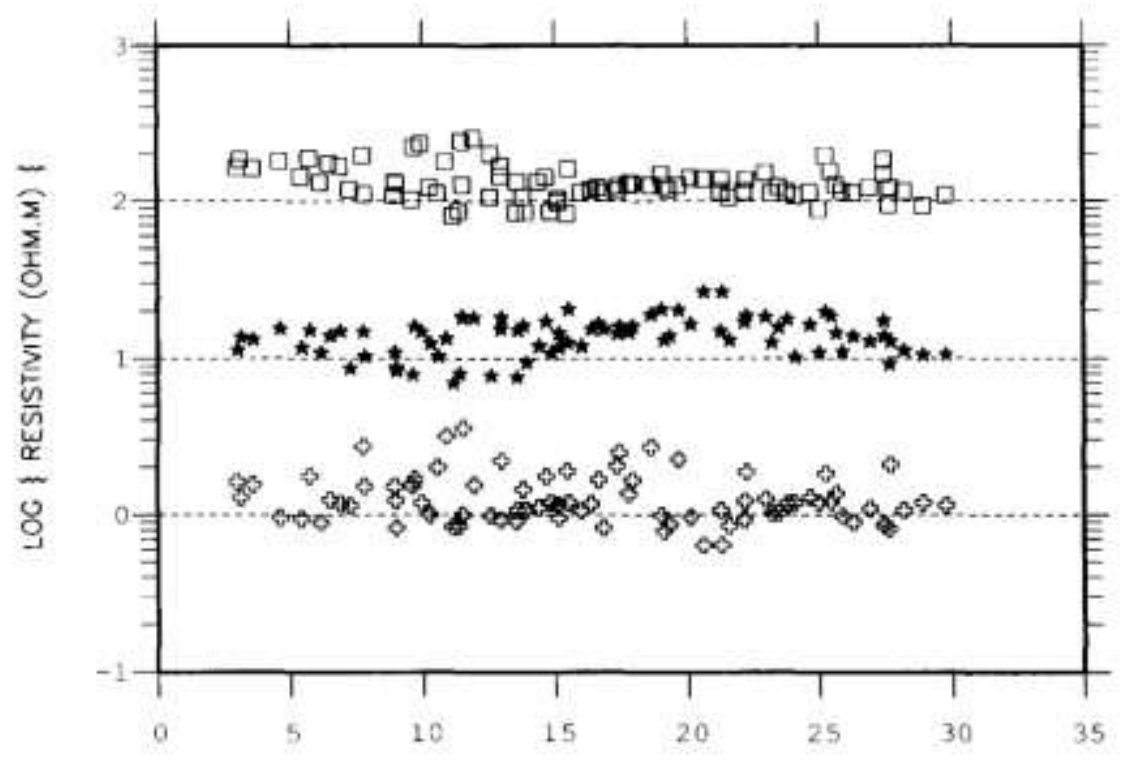

RADIAL. DISTANCE (KM)

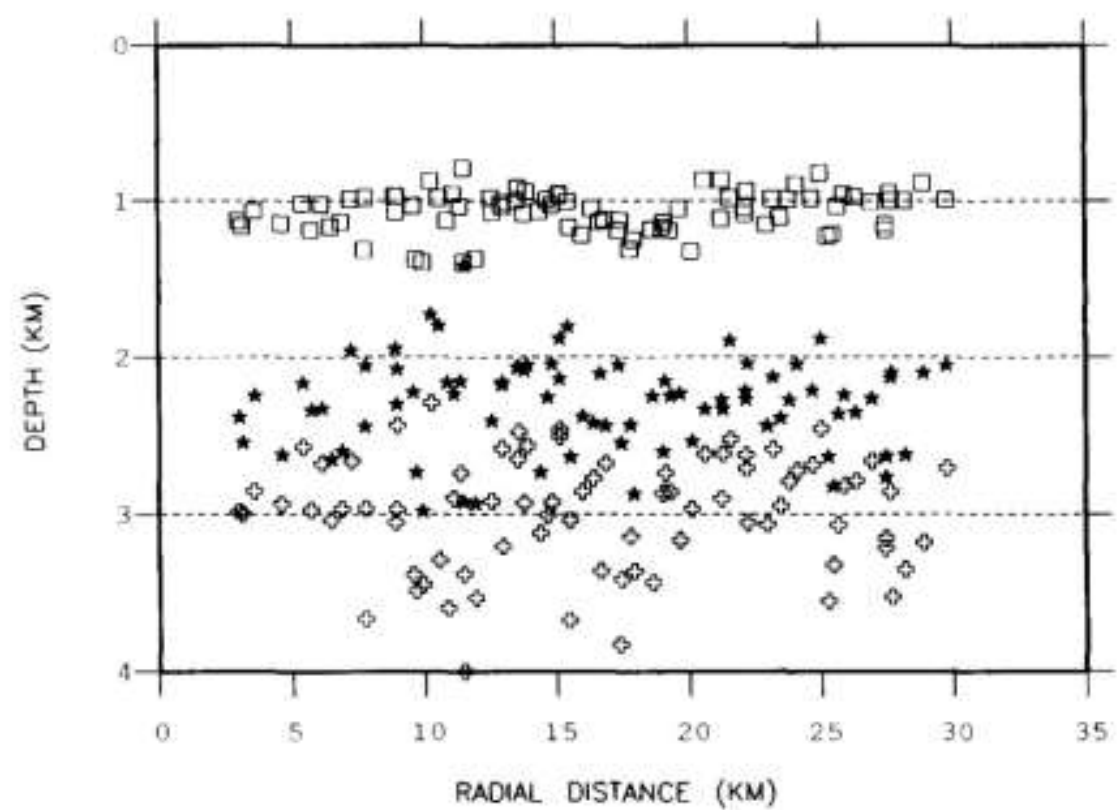

Fig. 10, 4 layer inversion results of first 3 layer parameters (resistivity and depth) obtained using the diamond averaged, invariant data at all 84 sites. The results are plotted as a function of radial distance from the array origin (67,70) of Fig. Ib. Layer 1 results (open circles), layer 2 results (solid stars), layer 3 results (open crosses). a. Upper diagram. Resistivities shown using a logarithmic scale. b. Lower diagram. Depths shown using a linear scale. 


\section{4-LAYERED INVERSION RESULTS \\ LAYER DEPTHS $1.2 \& 3$}

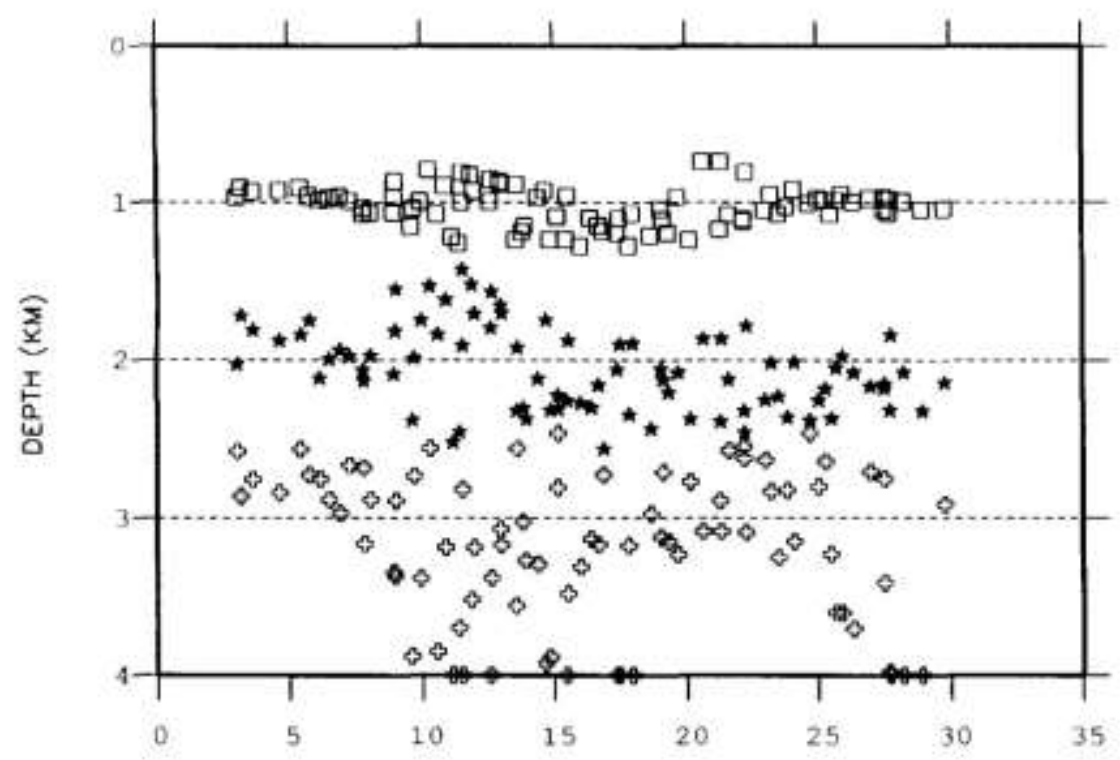

RADIAL. OISTANCE (KM)

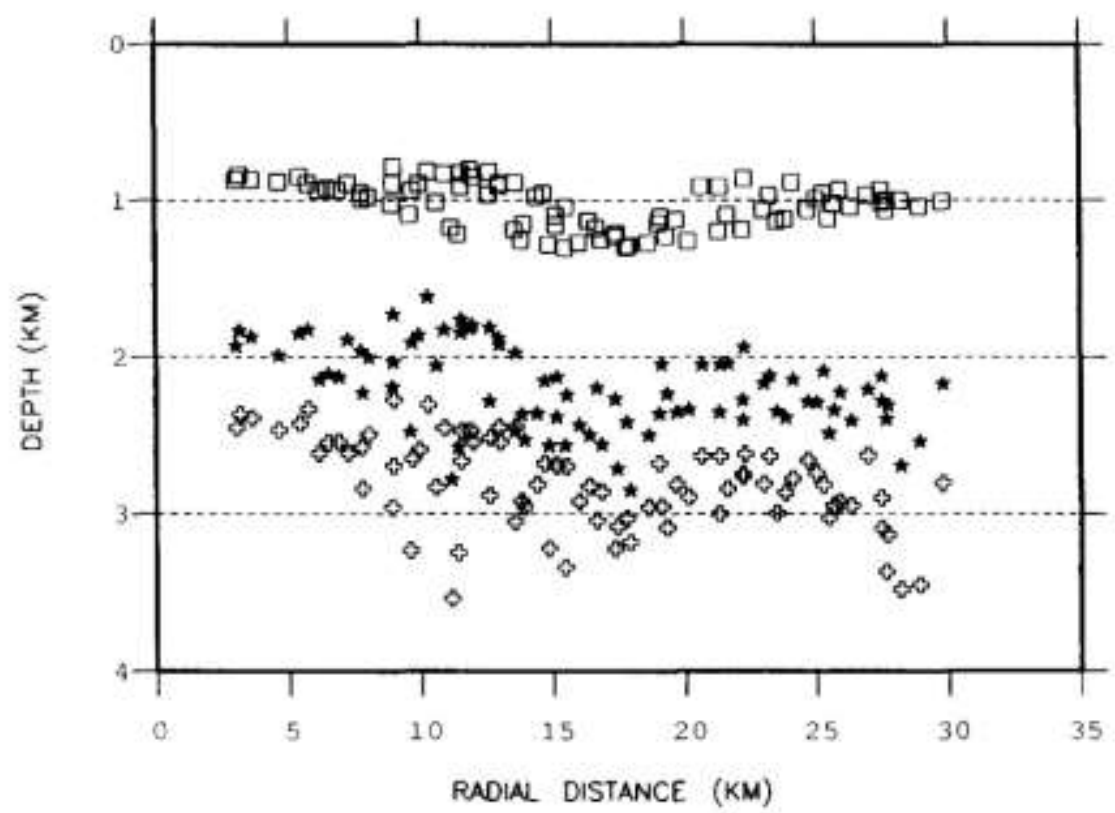

Fig. 11. 4 layer inversion results of 3 layer depths as per Fig. 10 but data have been inverted using phase alone and constraining one or more layers to have a uniform resistivity. a. Upper diagram. 1 layer constraint. Resistivity of layer 1 fixed at $125 \Omega \cdot \mathrm{m}$. b. Lower diagram. 3 layer constraints. Resistivities of layers fixed at $125 \Omega \cdot \mathrm{m}($ laycr 1 ), 14.4 $\Omega \cdot \mathrm{m}$ (layer 2 ) and $1.19 \Omega \cdot \mathrm{m}$ (layer 3 ). 
problem is non-linear. As noted previously, the assessments of static distortion alone can only be reasonably undertaken using a highly resolved layer parameter that is involved in only a marginal degree of equivalencing. Here, since the resistivity of layer 1 is being used as a constraint, the depth of layer 1 is considered.

The spatial $(x, y)$ maps of the estimates of the depth to base of layer 1 (equivalent to thickness) of Figs. 10b and $11 \mathrm{a}, \mathrm{b}$ are shown in sequence in Fig. 12. The chosen contour inter$\mathrm{val}$ is $100 \mathrm{~m}$ so the displays attempt to indicate the difficulties of estimating depth to about $10 \%$ (at a depth of $1 \mathrm{~km}$ ) in the presence of static distortion. The map of Fig. 12a is produced by unconstrained inversion of the joint apparent resistivity/phase data. It is therefore possible that these estimates contain errors due to "residual" distortion offsets. The question posed is whether the introduction of a fixed layer resistivity ( 1 layer in Fig. 12b or 3 layers in Fig. 12c) and the use of phase-alone inversion can improve our confidence in the depth estimation procedure.

A common feature of all three maps is the central maximum with a NNW trend to the north and a splay to the south and south-east. We have a high degree of confidence that this feature is broadly correct. The unconstrained inversion map of Fig. 12a contains some minor and isolated features and two regions of thickening, in the NW and SE corners, which are not found in the constrained (phase-alone) inversion maps. Our interpretation would be that these are likely to be due to residual distortion offsets which have influenced the joint apparent resistivity/phase inversions. They are unlikely to be correct. The two constrained maps of Figs. $12 \mathrm{~b}$ and $12 \mathrm{c}$ display a high degree of correspondence apart from the feature representing extreme thinning on the northern edge of the array (Fig. 12b). This degree of thinning is not found in the other two maps and is therefore suspect. Following this line of reasoning it appears appropriate to adopt the common features as being "best" estimates.
Without undertaking a formal correspondence analysis, it appears that the isopach map of Fig. $12 \mathrm{~b}$ appears to represent a map with estimates which provide the least conflict.

Thus far the control provided by the well-log in the vicinity of sites 8 and 10 has not been reassessed. A comparison of the models provided by the invariant data of method 2 (diamond-averaging alone) was shown in Fig. 2 and the poor estimation of the depth of the basalt layer was noted. In contrast to the results shown in Fig. 2, the 4 layer models obtained at the two sites using phase-only inversion and a single (layer 1) resistivity constraint were highly consistent. The model estimates of the depth to the base of the basalt layer were 1239 $\mathrm{m}$ at site 8 and $1238 \mathrm{~m}$ at site 10 and thus agree very well with the well-log estimate of $1230 \mathrm{~m}$.

The above results all suggest that diamondaveraged apparent resistivity data still contain some residual offsets due to static distortion, particularly in the NW and SE corners. This could be anticipated given the pervasive nature of the distortion encountered. The results of all the assessments suggest that the least-restrictive constraint, that of assuming a uniform resistivity for the basalt cover (layer 1 ), can provide an improvement in the removal of the unknown levels of static distortion contaminating the sounding data. Where well-log control exists, this method provides accurate estimates of the depth of the main resistivity interface of the vertical section.

When layered structural assessments are required the simplest procedure is to undertake phase-only data inversions with a common layer resistivity constraint for all soundings. As noted by Jones (1988), it is also possible to correct the apparent resistivity sounding data using the static shift factors $D_{x}(x, y), D_{y}(x, y)$ determined at each site. These may be estimated since they relate a particular best-fitting estimate of a layer parameter with a corresponding "true" estimate. For the procedure adopted the "true" estimate is simply the constrained layer parameter. In our case both $D_{x}(x, y)$ and 

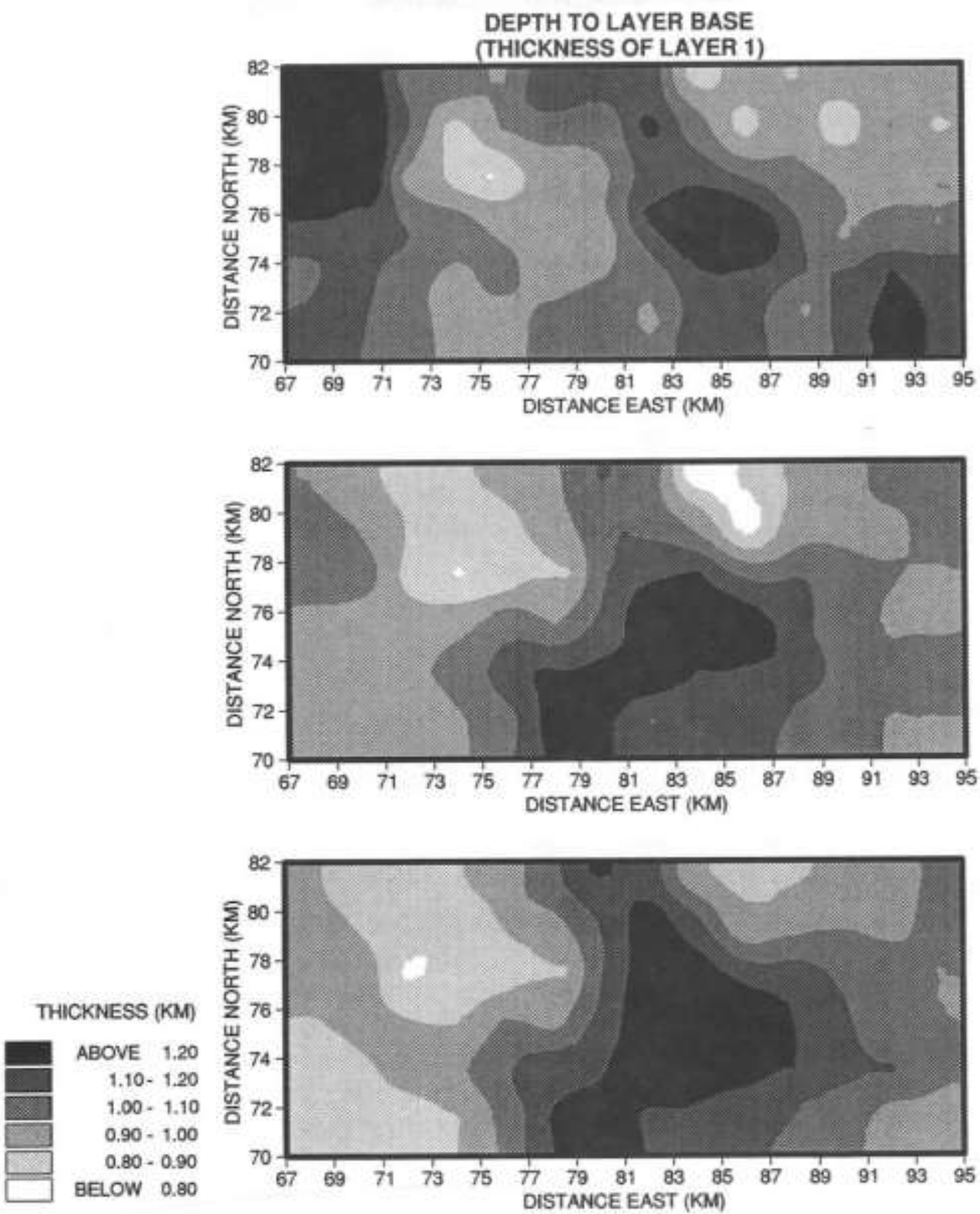

Fig. 12. Comparison of best-fitting estimates of depth (equivalent to thickness) of layer 1 obtained by different methods. Results shown in plan view, contour interval $100 \mathrm{~m}$. Upper diagram are results obtained using diamond averaged data and inverting apparent resistivity and phase jointly (method 2). Centre diagram shows results obtained by phase only inversion with resistivity of layer 1 fixed (method 3 ). Lower diagram shows results obtained by phase only inversion with resistivity of first 3 layers fixed (method 3 ).

$D_{y}\{x, y)$ were estimated for each sounding using the best-fitting estimates of the resistivity of layer 1 from phase-only data inversion. The exercise of performing data correction, form- ing the invariant and performing data inversion using apparent resistivity (corrected) and phase jointly produced an isopach surface equivalent to that shown in Fig. 12b. 


\section{Conclusions}

Using data from a detailed grid survey, three methods for the removal of static distortion have been assessed and compared. Assessments can be undertaken in both data and model spaces. The data obtained by the Clevelandia survey resolve 4 geoelectric units which comprise the flood basalts and two distinct sedimentary sequences, the lower of which is a highly conducting (about $1 \Omega \cdot \mathrm{m}$ ) formation. The underlying half-space, at a depth of about $3 \mathrm{~km}$, conforms to electrical basement.

The three methods considered were (1) individual curve shifting, (2) statistical (spatial) averaging, and (3) parameter constraints. The first method involves a constraint that is assessed using the data (not model) statistics. It was noted however that the use of the same value of apparent resistivity at a single frequency across the whole area effectively assumes that a uniform layer underlies the survey area, at a fixed depth. This is, in effect, a very restrictive constraint to apply. Since the response due to such a conceptual layer will encompass a finite bandwidth, the applicability of the method may be better subsumed by the techniques incorporated in method 3.

The second method involves no formal constraint but relies on the random nature of distortion offsets. This is a realistic assumption given the high wavenumber contribution attributable to near-surface inhomogeneities and the fact that our sounding centres are spaced at $2 \mathrm{~km}$ intervals. The technique involves an inevitable loss of spatial resolution whose severity is survey dependent. The results obtained with our data indicate that the method is more optimistic than rigorous when static distortion is pervasive.

In method 3, only the statistical behaviour of highly-resolved model parameters can be realistically assessed. In cases, as here, where a uniform resistivity can be assumed for a given layer, best-fitting models can be obtained using the phase data alone. In these circumstan- ces, the apparent resistivity data, which may contain unknown distortion offsets, are not included in model construction. The method allows the depths to all interfaces to be modelled and structural assessments are therefore unimpaired. The geoelectric models resulting from this approach were found to provide accurate estimates of the depth to the main resistivity interface (the base of the basalt cover) in the vicinity of a control well.

\section{Acknowledgements}

We thank Dr. F. Nepomuçenco of Petrobrás Research Center for supporting this work. We also thank Gaston Fischer for providing the algorithm code used in the inverse model assessments of the Clevelandia data. This paper is published with the approval of the Director, British Geological Survey (NERC).

\section{References}

Beamish, D., 1990. A deep geoelectric survey of the Carnmenellis granite. Geophys. J. Int., 102: 679-693.

Beamish, D. and Travassos, J.M., 1992a. The use of the $\mathrm{D}^{+}$solution in magnetotelluric interpretation. J. Appl. Geophys., 29: 1-19.

Beamish, D. and Travassos, J.M., 1992b. A study of magnetotelluric static distortion in the context of intrusive volcanism: Geophys. Prospect: in press.

Berdichevsky, M.N. and Dmitriev, V.I., 1976. Basic principles of interpretation of magnetotelluric sounding curves. In: A. Adam (Editor), Geoelectric and geothermal studies. KAPG Geophys. Monogr. Akad. Kiado, 165-221.

Berdichevsky, M.N., Vanyan, L.L. and Dmitriev, V.I., 1989. Methods in the U.S.S.R. to reduce near-surface inhomogeneity effects on deep magnetotelluric sounding. Phys. Earth Planet. Inter., 53: 194-206.

Bostick, F.X., 1986. Electromagnetic array profiling. 56th Annu. Int. Mtg., Soc, Explor. Geophys., Exp. Abstr., 60-61.

Clay, C.S. and Hinich, M.J., 1981. Estimating the earth's impedance function when there is noise in the electric and magnetic signals In: D.F. Findley (Editor), Applied Time Series Analysis, II. Academic Press, New York, pp. 189-210.

Fischer, G. and Le Quang, B.V., 1981. Topography and minimization of the standard deviation in one-dimen- 
sional magnetotelluric modelling. Geophys. J. R. Astron. Soc., 67: 279-292.

Jiracek. G.R., 1990. Near-surface and topographic distortions in electromagnetic induction. Surv. Geophys., 11: 163-203.

Jones, A.G., 1988. Static shift of magnetotelluric data and its removal in a sedimentary basin environment. Geophysics, 53: 967-978.

Jupp, D.L.B. and Vozoff, K., 1975. Stable iterative methods for the inversion of geophysical data. Geophys. J. R. Astron. Soc., 42: 957-976.

Larsen, J.C., 1977. Removal of local surface conductivity effects from low frequency mantle resporse curves. Acta Geodaet., Geophys. Montanist., Acad. Sci. Hung., 12: 183-186.

Oldenburg, D.W., 1990. Inversion of electromagnetic data: an overview of new techniques. Surv. Geophys., 11: 231-270.

Park, S.K., 1985. Distortion of magnetotelluric sounding curves by three-dimensional structures. Geophysics, 50: 785-797.

Pellerin, L. and Hohmann, G.W., 1990. Transient electromagnetic inversion: a remedy for magnetotelluric static shifts. Geophysics, 55: 1242-1250.

Raiche, A.P., Jupp, D.L.B., Rutter, H. and Vozoff, K.,
1985. The joint use of coincident loop transient electromagnetic and Schlumberger sounding to resolve layered structures, Geophysics, 50: 1618-1627.

Stanley, W.D., Saad, A.R. and Ohofugi, W., 1985, Regional magnetotelluric surveys in hydrocarbon exploration. Bull. AAPG, 69:346-360.

Sternberg, B.K., Washburne, J.C. and Anderson, R.G., 1985. Investigation of MT static shift correction methods. 55th Annu. Int. Mtg., Soc. Explor. Geophys., Exp. Abstr., 264-267.

Sternberg, B.K., Washburne, J.C. and Pellerin, L., 1988. Correction for the static shift in magnetotellurics using transient electromagnetic soundings. Geophysics, 53: 1459-1468.

Wannamaker, P.E., Hohmann, G.W. and Ward, S.H., 1984. Magnetotelluric responses of three-dimensional bodies in layered earths. Geophysics, 49; 1517-1533.

Weidelt, P., 1972. The inverse problem of geomagnetic induction. Z. Geophys., 38: 257-289.

Zalán, P.V., Conceiçáo, J.C.J., Wolff, S., Astolfi, M.A.M., Vieira, V.T., Santos Neto, E.V., Cerqueira, J.R., Zanoto, O.A. and Paumer, M.L., 1986. Arialise da Bacia do Paraná, GT-0s-009/85. PETROBRÁS/DEPEX, Rio de Janciro (unpubl.). 